Physics

Physics Research Publications

\title{
Astability and negative differential resistance of the Wigner solid
}
G. A. Csathy
D. C. Tsui
L. N. Pfeiffer
K. W. West

This paper is posted at Purdue e-Pubs.

http://docs.lib.purdue.edu/physics_articles/868 


\title{
Astability and Negative Differential Resistance of the Wigner Solid
}

\author{
G. A. Csáthy, ${ }^{1} *$ D. C. Tsui, ${ }^{1}$ L. N. Pfeiffer, ${ }^{2}$ and K. W. West ${ }^{2}$ \\ ${ }^{1}$ Department of Electrical Engineering, Princeton University, Princeton, New Jersey 08544, USA \\ ${ }^{2}$ Bell Labs, Lucent Technologies, Murray Hill, New Jersey 07974, USA
}

(Received 2 November 2005; published 7 February 2007)

\begin{abstract}
We report spontaneous narrow band oscillations in the high field Wigner solid. These oscillations are similar to the recently seen and yet unexplained oscillations in the reentrant integer quantum Hall states. The current-voltage characteristic has a region of negative differential resistance in the current biased setup and it is hysteretic in the voltage biased setup. As a consequence of the unusual breakdown, the oscillations in the Wigner solid are of the relaxation type.
\end{abstract}

DOI: 10.1103/PhysRevLett.98.066805

PACS numbers: 73.40.- c, 73.20.Qt, 73.63.Hs

The strong Coulomb interaction in two-dimensional electron gases (2DEG) subjected to magnetic fields $B$ leads to the formation of remarkable many-body ground states. An example is the family of electron solids which has recently been shown to be quite numerous [1-12]. The high field insulating phase (HFIP) and its close relative the reentrant insulating phase (RIP) [1-5] have been associated with the Wigner solid (WS), a periodic crystal of electrons [13]. WS has also been found close to integer Landau level filling factors $\nu$ [6]. The most recently discovered solids are the insulating phases in high Landau levels. It is thought that the reentrant integer quantum Hall states (RIQHS) are exotic solids similar to the WS but with the freedom of more than one electron per unit cell and the electronic stripe phases are anisotropic solids akin to the nematic phase of liquid crystals [7-9].

The $B$-field induced electron solids share many properties such as nonlinear dc current-voltage characteristics ( $I-V$ characteristic) with a well defined threshold for conduction [2-4] and sharp resonances in the microwave spectrum [4-6]. In particular, the RIQHS was also found to exhibit these transport signatures $[10,11]$. The RIQHS, however, appears to be unique among the electron solids since it is the only one developing spontaneous narrow band oscillations under dc biasing [12]. Because of the increasing oscillation frequency with an increasing bias current [12], the oscillations in the RIQHS were suggested to be akin to the washboard oscillations found in conventional charge density waves (CDW) [14]. The frequencies of the RIQHS in the $\mathrm{kHz}$ range are, however, orders of magnitude lower than the expected washboard frequencies [12]. These oscillations remain unexplained to date.

In this Letter we report the observation of spontaneous narrow band oscillations in the high field WS which are similar to those in the RIQHS [12]. Unlike the RIQHS [7], the WS conducts through a single channel. Taking advantage of this property we formulate a simple model that explains our observations. We find that the WS exhibits nonlinear $I-V$ characteristics that are fundamentally different than the earlier reported ones [2-4] and that due to these unusual $I-V$ characteristics the oscillations in the WS are not of the washboard type but are relaxation oscillations.

Figure 1(a) shows the diagonal resistance $R_{x x}$ of an exceptional 2DEG confined to a GaAs/AlGaAs quantum well and which has an areal density of $4.8 \times 10^{10} \mathrm{~cm}^{-2}$ and a mobility of $13 \times 10^{6} \mathrm{~cm}^{2} /$ Vs. $R_{x x}$ is measured in a four wire configuration using a small signal ac excitation. The hashed regions on the two sides of the $\nu=1 / 5$ mark the HFIP and the RIP, the two phases identified with high field WS [1-5].

Using the $I$-biasing setup, a two-lead dc biasing shown in Fig. 1(b), an interesting temporal behavior develops. The simultaneously measured voltage $V$ and $I$ waveforms are shown in Fig. 2. The sample is held at $B=11 \mathrm{~T}$ and $54 \mathrm{mK}$ and there is a $V_{b}=225 \mathrm{mV}$ battery voltage applied
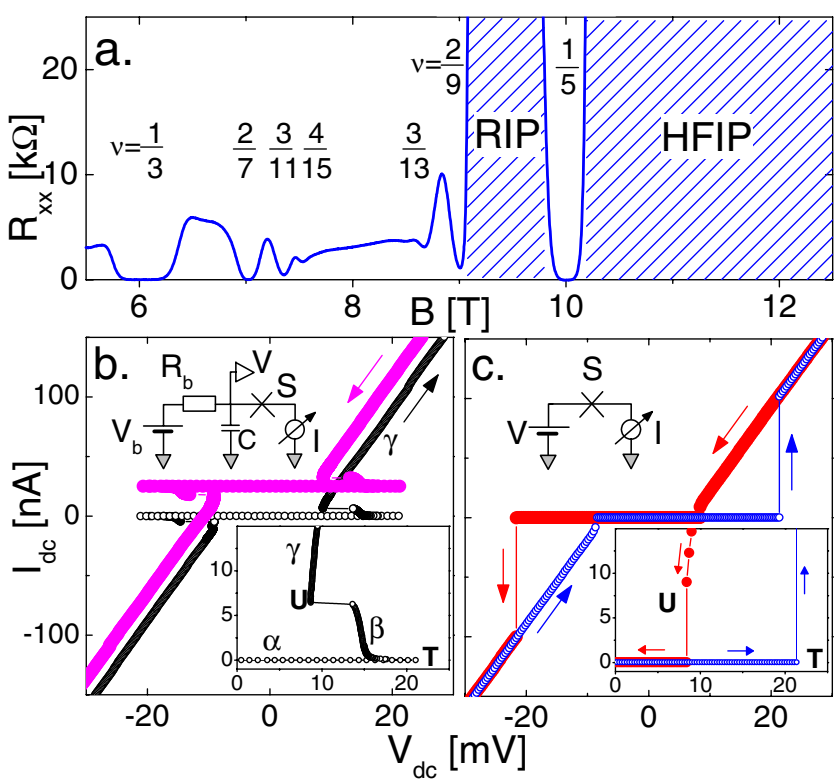

FIG. 1 (color online). Panel (a) shows the diagonal resistance $R_{x x}$ as a function of $B$ field at $38 \mathrm{mK}$. Lower panels show the two biasing circuits used and the corresponding $I-V$ characteristics for the sample $S$ held at $54 \mathrm{mK}$ and at $11 \mathrm{~T}$. The curve of decreasing current (full symbols) of panel (b) is offset by $25 \mathrm{nA}$. Insets show a magnified view of the $I-V$ characteristics. 


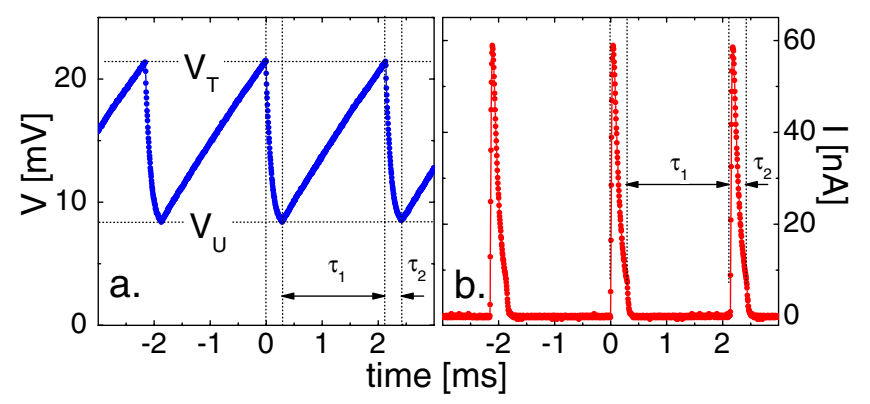

FIG. 2 (color online). $V$ and $I$ waveforms of the $I$-biased sample at $V_{b}=225 \mathrm{mV}$ bias. The sample is at $B=11 \mathrm{~T}$ and $T=54 \mathrm{mK}$.

to it through the $R_{b}=50 \mathrm{M} \Omega$ biasing resistor. The bandwidth for $V$ and $I$ is limited by the preamplifiers to $700 \mathrm{kHz}$ and $8 \mathrm{kHz}$, respectively. Both $V$ and $I$ oscillate in phase at the frequency of $f=0.465 \mathrm{kHz}$. The quality factor is 400 and in various cooldowns we measured values as high as 1000 . The two waveforms are quite different. $V$ has slowly increasing regions which appear to be exponential and which alternate with quick drops. Correlated with this behavior, $I$ vanishes then it has a narrow spike.

The dc $I-V$ characteristic shown in Fig. 1(b) was obtained using the same $I$-biased circuit as the one used to detect the oscillations but, as customary for de $I-V$ characteristics, we limit the measurement bandwidth with integrators having their time constant $0.3 \mathrm{~s}$. We thus record $I_{\mathrm{dc}}$ and $V_{\mathrm{dc}}$, the temporal averages of $I$ and $V$. Branch $\alpha$ of the $I-V$ characteristic, which carries virtually no current and $\gamma$ which supports conduction are commonly found in insulators. To our surprise we find a third branch labeled $\beta$ which exhibits negative differential resistance (NDR).

We examine next the relationship of the oscillations and the $I-V$ characteristic. Oscillatory behavior similar to that in Fig. 2 develops when the biasing point, i.e., the crossing point of the load line and the $I-V$ characteristic, is on branch $\beta$. In contrast, biasing anywhere on branches $\alpha$ and $\gamma$ leads to the development of a steady state with no change of $V$ or $I$ with time. We conclude therefore that branch $\beta$ exhibiting NDR is a result of the temporal averaging of the oscillations developed. Figure 3 summarizes the bias dependence of the oscillations. The amplitude of both the $V$ and $I$ waveforms does not change with bias. Furthermore, a comparison of Figs. 1(b) and 2(a) reveals that the extremes of the bias-independent amplitude of the $V$ waveform coincide with $V_{U}$ and $V_{T}$, the voltages of the endpoints of the branches $\alpha$ and $\gamma$ of the $I-V$ characteristic. We find that $f$ is slightly sublinear and increasing function of the bias current $I_{\mathrm{dc}}$. The width $\tau_{2}$ of the current spikes depends only weakly on the bias. The oscillations are still well developed at $I_{\mathrm{dc}}=5.7 \mathrm{nA}$ but they disappear for $I_{\mathrm{dc}}>5.8 \mathrm{nA}$. In the narrow current range between the above two values $V$ and $I$ have burstlike behavior (not shown) that is nonperiodic and that is strongly influenced by fluctuations in the electrical and thermal environment of the sample.
Besides $I$ biasing, $V$ biasing is another means of measuring insulators such as the WS. The circuit and the $I-V$ characteristic obtained using the same pair of ohmic contacts are shown in Fig. 1(c). The $V$-biased $I-V$ characteristic has two linear branches. When the voltage is increased the current stays virtually zero as long as the bias does not exceed the threshold value $V_{T}$. As the voltage is further increased through the threshold electrical breakdown occurs when the current jumps from $3 \mathrm{pA}$ to $100 \mathrm{nA}$. No intermediate values of $I$ are allowed. Once the voltage is decreased from a starting point beyond the threshold, $I$ takes nonzero values by decreasing until it suddenly jumps to zero at $V_{U}$ substantially less than $V_{T}$. Superimposing Figs. 1(b) and 1(c) (not shown), the linear branches of the two $I-V$ characteristics taken with the two different circuits perfectly overlap. An exception is the region of the $I-V$ characteristics which has NDR for $I$ biasing and which is hysteretic for $V$ biasing. Using the $V$-biased configuration we detect no oscillations at any of the $B$ fields.

The extremely sharp onset of conduction and the hysteresis for the $V$-biased setup, the NDR and the generation of narrow band oscillations for the $I$-biased setup are present for all pairs of contacts but only in the WS phase, i.e., for $9.30 \leq B \leq 9.46 \mathrm{~T}$ and $10.40 \leq B \leq 14 \mathrm{~T}$. We therefore think that these transport properties are not due to contact effects.

Next we show that the ever-present cable capacitance $C$ is essential for the development of the oscillations. As seen in Fig. 2(b), there are time intervals when the current flowing through the sample is zero while the current through $R_{b}$ is $4.5 \mathrm{nA}$. Kirchhoff's law of current conservation thus can only be satisfied if nonzero current flows

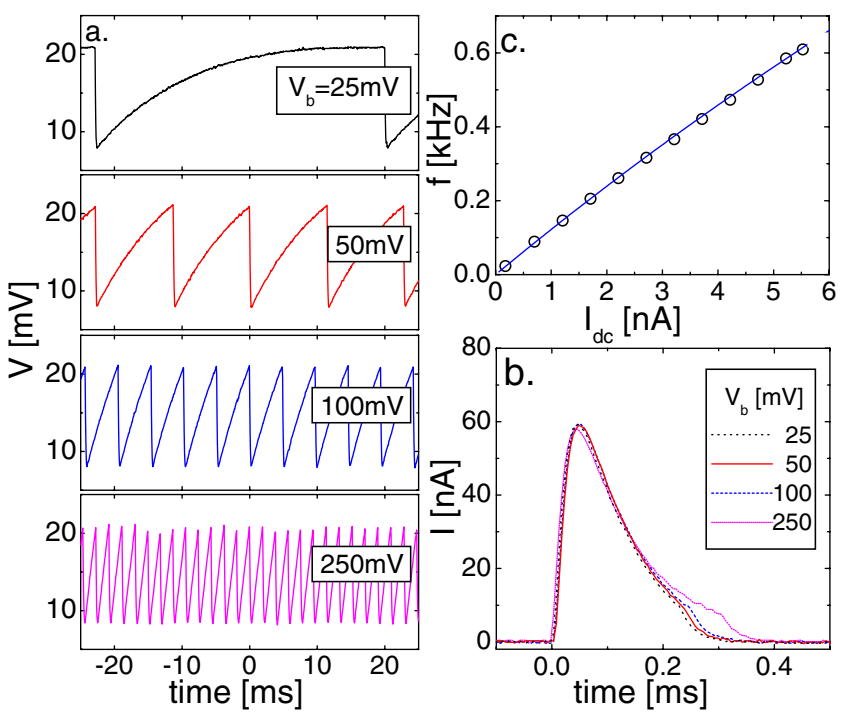

FIG. 3 (color online). The dependence of the $V$ [panel (a)] and the $I$ oscillations [panel (b)] and of the oscillating frequency [panel (c)] on the biasing conditions. The line in panel (c) is the prediction of the model described in the text and it has no adjustable parameters. The sample is at $B=11 \mathrm{~T}$ and $T=$ $54 \mathrm{mK}$. 
through $C$. Analyzing the $I$-biased circuit one finds that when the load line crosses any or both of branches $\alpha$ and $\gamma$ of the $I-V$ characteristic there is a steady state developing. However, when the load line crosses only the NDR region of the $I$-biased $I-V$ characteristic or, equivalently, does not cross any of the branches of the $V$-biased $I-V$ characteristic, the circuit is astable, i.e., it exhibits temporal oscillations between two predetermined states. Indeed, connecting the battery the sample does not conduct and $C$ charges up towards $V_{b}$ with the time constant $R_{b} C$. When the voltage $V$ on the sample reaches its threshold value $V_{T}$, however, the sample conducts and $C$ quickly discharges through the sample. The discharge continues until $V$ drops to $V_{U}$. At this point the charging process begins anew leading to cyclical variations of $V$ and $I$. Such oscillations are known as relaxation oscillations and the circuit is called astable multivibrator or Schmitt trigger [15].

Relaxation oscillations are commonly observed in devices with NDR such as the tunnel diode, diac, and triac [16], in superconducting devices (SD) like the Josephson junction [17] and superconducting microbridges [18], and in CDW [19]. For all of the devices above oscillations develop when biasing in the NDR region, the amplitude of the oscillations is independent of the bias, and the oscillation frequency increases with increasing bias. They can be grouped according to the type of NDR: the $I-V$ characteristic of the WS shown in Fig. 1(b) is $S$-shaped and the NDR is said to be current controlled while the $I-V$ characteristic in the SD [17,18], for example, is $N$-shaped and its NDR is voltage controlled [16]. The oscillations in the devices with $S$-shaped $I-V$ characteristics develop only when samples are biased with a large impedance source and the external reactance is a capacitance, while those in devices with $N$-shaped $I$ - $V$ characteristics must be biased with a low impedance source and there must be an inductance present. The $I-V$ characteristics of these two groups can be mapped into each other by exchanging the axes. This symmetry is also reflected in the temporal waveforms; the voltage waveform of the WS is qualitatively similar to that of the current waveform of the SD $[17,18]$.

The simplicity of the $I-V$ characteristic allows for a quantitative description. We model the branches by $I=0$ for $V<V_{T}$ and $I=\left(V-V_{0}\right) / r$ for $V>V_{U}$. Here $r$ is the differential resistance of the current carrying branch and $V_{0}$ is where the extension of this branch crosses the $V$ axis. Solving the equations of motion the charging and discharging time of the capacitor obtained in the large $R_{b}$ approximation are $\tau_{1} \simeq C\left(V_{T}-V_{U}\right) / I_{\mathrm{dc}}$ and $\tau_{2} \simeq$ $r C \ln \left[\left(V_{T}-V_{0}\right) /\left(V_{U}-V_{0}\right)\right]$, respectively. The frequency of the oscillations $f=1 /\left(\tau_{1}+\tau_{2}\right)$ is thus a function of quantities characterizing both the $I-V$ characteristic of the sample as well as the external biasing circuitry. The parameters $V_{T}=21.4 \mathrm{mV}, V_{U}=8.4 \mathrm{mV}, V_{0}=6.5 \mathrm{mV}$, and $r=144 \mathrm{k} \Omega$ extracted from the $I-V$ characteristic of Fig. 1 and the cable capacitance of $C \simeq 610 \mathrm{pF}$ yield $\tau_{2}=$ $0.18 \mathrm{~ms}$ and a current dependence of $f$ shown as a continuous line in Fig. 3(c). These results are in excellent agreement with our data. As a test, we vary the external circuit elements at a fixed $225 \mathrm{mV}$ bias. Results are shown in Fig. 4. In agreement with our model the frequency is found to be inversely proportional to $C$ and independent of $R_{b}$ at large $R_{b} s$.

We focus next on the unusual $I-V$ characteristics of the WS which, as shown, are essential in generating the oscillations. Earlier measured $I-V$ characteristics are nonlinear and the transition from zero to finite current is smooth [24]. In contrast, the $I-V$ characteristics of the WS are qualitatively different as they exhibit a sharp onset of conduction and hysteresis. One scenario that leads to such behavior is the avalanche breakdown due to impact ionization of shallow impurities in $n$-doped pure and compensated bulk GaAs [20]. Such a process in our sample, however, is very unlikely because of the extremely high purity of the quantum well. Furthermore, the magnitude of the breakdown electric field and its $B$ dependence, as well as the $B$ dependence of the hysteresis loop are inconsistent with those at impact ionization [20]. We thus conclude that the transport signatures we observe are fundamental properties of the high field WS at low $T$ in a sample of high quality.

The breakdown of the WS has been interpreted as being due to the depinning of the WS [2-4]. According to this picture a perfectly periodic WS is deformed and broken up into domains when experiencing the quenched disorder of the host semiconductor [21]. Once subjected to a small external electric field these domains are deformed but remain pinned by the disorder hence the insulating behavior. As the electric field is increased beyond a threshold, sliding of the solid commences. The smooth $I-V$ characteristics reported earlier are inconsistent with such a model of breakdown as lowering the external field just below the threshold should not pin the WS. This is because an already moving WS can gain energy from the electric field to overcome pinning. Such an energy gain cannot occur in a pinned WS. Hence the sharp steplike discontinuity and hysteresis of the $I-V$ characteristic we report are consistent, for the first time, with the transition from a pinned to a

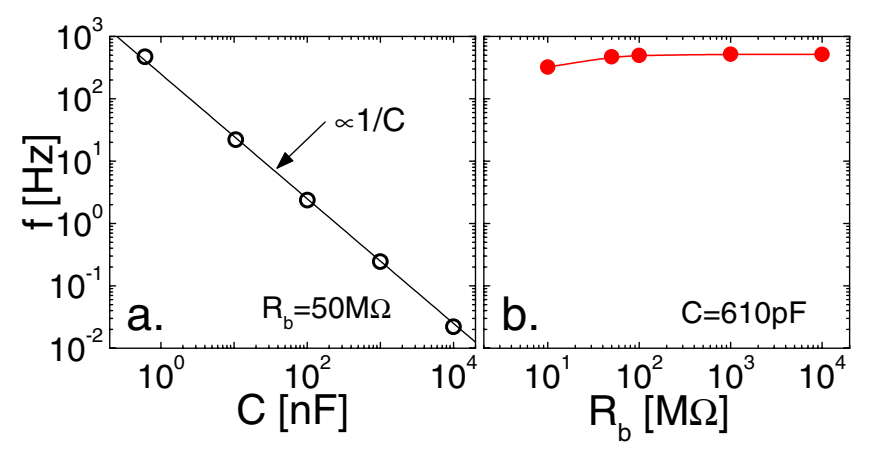

FIG. 4 (color online). The dependence of the frequency of oscillations on the capacitance to ground $C$ at $R_{b}=50 \mathrm{M} \Omega$ [panel (a)] and on the biasing resistor $R_{b}$ at $C=610 \mathrm{pF}$ [panel (b)] at $225 \mathrm{mV}$ bias. 
sliding WS. From the theoretical point of view the $I-V$ characteristic of a prototypical elastic medium in the presence of quenched disorder is model dependent and can be hysteretic within the framework of models which consider phase slips between the domains [22], incorporate viscosity [23], and include the effect of built-up stress on the underdamped motion of the domains [24].

The argument and theories above do not explicitly include hot electron effects. A model for thermal runaway [25] was proposed for the hysteretic $I-V$ characteristics observed for the integer quantum Hall states [26]. We first consider the possibility of self-heating below threshold, i.e., on the branch of the $I-V$ characteristic that carries virtually no current. $I$ reaches its maximum value of $3 \mathrm{pA}$ at $V_{T}$ where the dissipated power is about $60 \mathrm{fW}$. This current could be due to defects such as interstitial electrons moving in a Bloch energy band created by the periodic potential of the electrons of the WS. In the metallic state $60 \mathrm{fW}$ does not cause self-heating [27], but the effect of such a dissipated power on the WS is not known. Nonetheless self-heating on this branch of the $I-V$ characteristic is unlikely since at a fixed $B$ the measured $V_{T}$ is not $T$ independent even at the lowest $T s$. We therefore think that below $V_{T}$ the WS is not destroyed by self-heating. After breakdown, however, the dissipated power soars 5 orders of magnitude. Such an increased power level could melt the WS but it is also possible that fast-moving charges reorder into a sliding WS. Reordering after depinning has been predicted and experimentally observed in the vortex lattice of type II superconductors [28] and it has been proposed in a theory for the classical WS [29].

To summarize, the $I-V$ characteristic of the high field WS is hysteretic in the $V$-biased setup and exhibits a region of NDR in the $I$-biased configuration. While these results cannot unambiguously identify the WS, they are consistent with and therefore strengthen the pinned WS interpretation. At special biasing conditions spontaneous $V$ and $I$ oscillations develop which are of the relaxation type. The oscillations arise from the switching between the pinned WS and a conducting state yet to be determined. Finally, we note that due to the different measurement setups a comparison of the results obtained by us and those for the RIQHS $[10,12]$ is not straightforward. Several properties of the two phases, however, show striking resemblance. For both electronic solids the $I-V$ characteristics are hysteretic, oscillations develop in the audio frequency range, the oscillation frequency increases with increasing bias, and the oscillation amplitude is independent of the bias.

This research was funded by the U.S. DOE and NSF. G. A. C. was supported on the DOE Grant No. DE-FG0298ER45683.

*Present address: Department of Physics, Purdue University, West Lafayette, Indiana 47907, USA.
[1] V. J. Goldman, M. Shayegan, and D. C. Tsui, Phys. Rev. Lett. 61, 881 (1988); R. L. Willett et al., Phys. Rev. B 38, R7881 (1988); H. W. Jiang et al., Phys. Rev. Lett. 65, 633 (1990).

[2] R. L. Willett et al., Phys. Rev. B 40, 6432 (1989); V. J. Goldman et al., Phys. Rev. Lett. 65, 2189 (1990); Y. P. Li et al., Phys. Rev. Lett. 67, 1630 (1991).

[3] H. W. Jiang et al., Phys. Rev. B 44, 8107 (1991).

[4] F. I. B. Williams et al., Phys. Rev. Lett. 66, 3285 (1991).

[5] M. A. Paalanen et al., Phys. Rev. B 45, 11342 (1992); L. W. Engel et al., Solid State Commun. 104, 167 (1997); P. D. Ye et al., Phys. Rev. Lett. 89, 176802 (2002); Y.P. Chen et al., Phys. Rev. Lett. 93, 206805 (2004); Y. P. Chen et al., Nature Phys. 2, 452 (2006).

[6] Y. Chen et al., Phys. Rev. Lett. 91, 016801 (2003); R. M. Lewis et al., Physica (Amsterdam) 22E, 104 (2004).

[7] M. P. Lilly et al., Phys. Rev. Lett. 82, 394 (1999); R. R. Du et al., Solid State Commun. 109, 389 (1999).

[8] J. P. Eisenstein et al., Phys. Rev. Lett. 88, 076801 (2002); J. S. Xia et al., Phys. Rev. Lett. 93, 176809 (2004).

[9] A. A. Koulakov, M. M. Fogler, and B. I. Shklovskii, Phys. Rev. Lett. 76, 499 (1996).

[10] K. B. Cooper et al., Phys. Rev. B 60, R11 285 (1999).

[11] R. M. Lewis et al., Phys. Rev. Lett. 89, 136804 (2002).

[12] K. B. Cooper et al., Phys. Rev. Lett. 90, 226803 (2003).

[13] E. P. Wigner, Phys. Rev. 46, 1002 (1934); Y. E. Lozovik and V. I. Yudson, JETP Lett. 22, 11 (1975); H. Fukuyama, P. M. Platzman, and P. W. Anderson, Phys. Rev. B 19, 5211 (1979).

[14] R. M. Fleming and C. C. Grimes, Phys. Rev. Lett. 42, 1423 (1979).

[15] See, for example, A.S. Sedra and K.C. Smith, Microelectronic Circuits (Oxford University, New York, 1998).

[16] S.M. Sze, Physics of Semiconductor Devices (John Whiley \& Sons, New York, 1981).

[17] F. L. Vernon and R. J. Pedersen, J. Appl. Phys. 39, 2661 (1968).

[18] W. J. Skocpol, M. R. Beasley, and M. Tinkham, J. Appl. Phys. 45, 4054 (1974); R. P. Huebner, J. Appl. Phys. 46, 4982 (1975).

[19] L. Mihály and G. Grüner, Solid State Commun. 50, 807 (1984).

[20] R. A. Reynolds, Solid-State Electron. 11, 385 (1968); K. Aoki, T. Kondo, and T. Watanabe, Solid State Commun. 77, 91 (1991).

[21] A. I. Larkin, JETP 31, 784 (1970); H. Fukuyama and P. A. Lee, Phys. Rev. B 17, 535 (1978).

[22] S. H. Strogatz et al., Phys. Rev. Lett. 61, 2380 (1988).

[23] M. C. Marchetti et al., Phys. Rev. Lett. 85, 1104 (2000); M. C. Marchetti et al., Phys. Rev. Lett. 91, 107002 (2003).

[24] J.M. Schwarz and D. S. Fisher, Phys. Rev. Lett. 87, 096107 (2001).

[25] S. Komiyama et al., Solid State Commun. 54, 479 (1985).

[26] G. Nachtwei, Physica (Amsterdam) 4E, 79 (1999).

[27] A. K. M. Wennberg et al., Phys. Rev. B 34, 4409 (1986).

[28] A. E. Koshelev and V. M. Vinokur, Phys. Rev. Lett. 73, 3580 (1994); U. Yaron et al., Nature (London) 376, 753 (1995).

[29] C. Reichhardt et al., Phys. Rev. Lett. 86, 4354 (2001). 